\section{POS0528 SCORES OF CARDIOVASCULAR RISK CALCULATORS AS PREDICTORS OF EARLY ATHEROSCLEROSIS IN PATIENTS WITH RHEUMATOID ARTHRITIS}

E. Gerasimova ${ }^{1}$, T. Popkova ${ }^{1}$, D. Gerasimova ${ }^{2}$, S. Glukhova ${ }^{3}$, E. Nasonov ${ }^{1,4}$, A. Lila ${ }^{1,5}{ }^{1}$ V.A. Nasonova Research Institute of Rheumatology, Systemic Rheumatic Diseases Laboratory, Moscow, Russian Federation; ${ }^{2}$ Sechenov First Moscow State Medical University (Sechenov University), Department of Organization and Economics of Pharmacy, Moscow, Russian Federation; ${ }^{3}$ V.A. Nasonova Research Institute of Rheumatology, Laboratories of Medical and Social Problems of Rheumatology, Moscow, Russian Federation; ${ }^{4}$ Sechenov First Moscow State Medical University (Sechenov University), Department of Rheumatology, Moscow, Russian Federation; ${ }^{5}$ Russian Medical Academy of Continuing Professional Education, Department of Rheumatology, Moscow, Russian Federation

Background: Rheumatoid arthritis (RA) is a systemic inflammatory disease leading to significant increase in cardiovascular morbidity and mortality. Development of cardiovascular diseases (CVD) in RA patients is associated with the accumulation of traditional risk factors and immunological disorders. Carotid Artery Doppler Ultrasound (DUS) Exam is the gold standard to identify early atherosclerosis. Objectives: To evaluate the cardiovascular risk and analyze its relationship with detection of early carotid artery atherosclerotic lesion in patients with rheumatoid arthritis (RA).

Methods: One hundred and nine RA patients (female/male 93/16), aged 45 to 60 without established CVD were included in the study. The median age was 52 [48; 54] years, duration of RA was 120 [36; 204] months, DAS28 was 4,7 $[3,5 ; 5,6]$ points. $54 \%(n=59)$ of patients received methotrexate (median dose 20 [15;27]mg/week), $12 \%$ - leflunomide (20 mg/day), $12 \%$ - sulfasalazine $(2000 \mathrm{mg} /$ day), $40 \%$ - glucocorticoids (median dose 4 [2;8]mg/day), $44 \%$ - non-steroidal anti-inflammatory drugs. None of study participants had biologics or statins in their therapeutic regiments at the time of inclusion. CVD risk was calculated with mSCORE, RRS, ASSIGN, QRISK3, ERS-RA scales and results of Carotid Artery DUS imaging in all patients.

Results: Traditional risk factors were highly among RA patients without CVD, including: arterial hypertension - in $73 \%$, dyslipidemia - $68 \%$, overweight - $53 \%$, family history of CVD - $43 \%$ and smoking - 39\% patients. A combination of three traditional risk factors was found in $60 \%$ of patients with RA, and combination of two - in $25 \%$. High risk was found in $5,5,14,6$, and $38 \%$ of patients according to mSCORE, RRS, ASSIGN, QRISK3, ERS-RA scales, respectively. Carotid atherosclerotic plaques were found in $30 \%$ of patients, more often in men (48\%) than in women $(28 \%, p=0,049)$, with similar prevalence regardless RA activity or stage od disease. Positive correlations of carotid intima-media thickness were established with scores of all CVD risk calculators: $m S C O R E(R=0,50)$, $R R S(R=0,40)$, ASSIGN $(R=0,40)$, QRISK3 $(R=0,36)$, ERS-RA $(R=0,26), p<0,05$ in all cases, as well as with age $(R=0,41 ; p=0,04)$, the level of total cholesterol $(R=0,23 ; p$ $=0,01)$, systolic blood pressure $(R=0,66 ; p=0,02)$, diastolic blood pressure $(R=$ $0,33 ; P=0,03)$, erythrocyte sedimentation rate $(R=0,26 ; p=0,04)$, IL-6 levels ( $R$ $=0,65 ; p=0,01)$. The sensitivity and specificity of the CVR algorithms in prognostication of atherosclerotic carotid artery lesions were 73 and $67 \%$ for mSCORE, 64 and $63 \%$ for RRS, 64 and $56 \%$ for ASSIGN, 73 and $49 \%$ for QRISK3, respectively, $p<0.05$ in all cases, 67 and $50 \%$ for ERS-RA, $p=0,06$.

Conclusion: RRS, mSCORE, ASSIGN, QRISK 3 calculators equally predicted the presence of carotid atherosclerotic plaques in RA patients. The optimal ratio of specificity and sensitivity is shown for the mSCORE scale. Stratification of CVR in RA patients should include assessment of the carotid intima-media thickness. mSCORE calculation and carotid intima-media thickness were the most informative methods identify CVR assessment in RA patients.

Disclosure of Interests: None declared

DOI: 10.1136/annrheumdis-2021-eular.1787

\section{POS0529 ANTI-TNF AND METHOTREXATE PROTECT AGAINST RHEUMATOID ARTHRITIS ATHEROSCLEROSIS}

M. Rojas-Giménez ${ }^{1}$, M. Á. Puche Larrubia', I. Gómez García', J. Calvo Gutierrez ${ }^{1}$, M. C. Ábalos-Aguilera ${ }^{2}$, A. Escudero Contreras ${ }^{1} .{ }^{1}$ Reina Sofia University Hospital, Cordoba, Maimonides Institute for Research in Biomedicine of Cordoba (IMIBIC), Córdoba University (UCO), Córdoba, Spain., Cordoba, Spain; ${ }^{2}$ Maimonides Institute for Research in Biomedicine of Cordoba (IMIBIC), Córdoba, Spain, Cordoba, Spain

Objectives: To analyze the effect of differents anti-rheumatic treatments on the carotid intima-medial thickness (cIMT) in patients with Rheumatoid Arthritis (RA). Methods: Controlled cross-sectional observational study of a cohort of patients with RA. 146 patients with RA (ACR/EULAR2010).cIMT was measured by semi-automatic carotid ultrasound.Other variables: atheroma plaques, bilateral plaques, DAS28-VSG, lipid metabolism, apoB, apoA1, apoB/apoA1, uric acid, homocysteine. Biological or conventional synthetic disease-modifying antirheumatic drug therapy (bDMARDs/csDMARDs), glucocorticoids. Descriptive, bivariate, Spearman's test and multivariate model were constructed to identify factors associated with cIMT.

Results: The baseline characteristics are shown in Table 1.Significant positive correlation was found between cIMT and apoB $(r=0.22 ; p=0.014)$, apoB/ apoA1 $(r=0.18 ; \quad p=0.044)$, cholesterol $(r=0.23 ; \quad p=0.006)$, triglycerides $(r=0,21$, $p=0.013)$, uric acid $(r=0.26 ; p=0.009)$, age $(r=0.65, p>0.001), \operatorname{RF}(r=0.16, p=0.05)$ homocysteine $(r=0.5, p<0.001)$, BMI $(r=0.19, p=0.023), \operatorname{CRP}(r=0.18, p=0.032)$ and negative correlation with anti-TNF $\alpha$ treatment time $(r=-0.20, p=0.02)$. Patients receiving bDMARDs alone or combined with csDMARDs had less CIMT than those taking only csDMARDs[median (IQR): 0.54 (0.50-0.63), 0.57(0.53-0.65) and $0.65(0.53-0.75) ; p=0.046]$.The factors associated with clMT were age $(B=$ $0.007, p<0.001)$,anti-tnf $\alpha$ treatment ever $(B=-0.06, p=0.027)$ and methotrexate treatment ever $(B=-0.07, p=0,08)$.

Table 1. Baseline characteristics

\begin{tabular}{|c|c|}
\hline Variables & RA $n=146$ \\
\hline \multicolumn{2}{|l|}{ Epidemiological characteristics and comorbidities } \\
\hline Age (years), mean (SD) & $55.9(13.1)$ \\
\hline Female sex; $\mathrm{n}(\%)$ & $112(76.7)$ \\
\hline Smoker, n (\%) & $30(21.6)$ \\
\hline Arterial hypertension, n (\%) & $45(30.8)$ \\
\hline Diabetes mellitus, $\mathrm{n}(\%)$ & $5(3.4)$ \\
\hline Previous Cardiovascular disease, n (\%) & $10(14.6)$ \\
\hline Hyperlipidemia, n (\%) & $46(31.5)$ \\
\hline $\mathrm{BMI}\left(\mathrm{kg} / \mathrm{m}^{2}\right)$, median $(\mathrm{IQR})$ & $26.8(23.4-29.7)$ \\
\hline \multicolumn{2}{|l|}{ Clinical-laboratory characteristics } \\
\hline Disease duration, years, median (IQR) & $6.77(2.2-14.2)$ \\
\hline Erosions, $\mathrm{n}(\%)$ & $53(36.6)$ \\
\hline RF positive, n (\%) & 119 (81.55) \\
\hline ACPA positive, $n(\%)$ & $123(84.2)$ \\
\hline CRP (mg/dl), median (IQR) & $4.8(1.6-11.5)$ \\
\hline ESR (mm/h), median (IQR) & $12(6-24)$ \\
\hline DAS28 at protocol, median (IQR) & $2.8(2.1-3.7)$ \\
\hline $\mathrm{HAQ}$, mean (SD) & $0.78(0.6)$ \\
\hline ApoB (mg/dl), mean (SD) & $86.8(21.8)$ \\
\hline ApoA1 (mg/dl), mean (SD) & $149.8(32.7)$ \\
\hline Cholesterol (mg/dl), mean (SD) & $198.7(37.3)$ \\
\hline $\operatorname{cLDL}(\mathrm{mg} / \mathrm{dl})$, mean $(\mathrm{SD})$ & $119.3(30.3)$ \\
\hline $\mathrm{cHDL}(\mathrm{mg} / \mathrm{dl})$, median (IQR) & $56(47-70)$ \\
\hline Triglycerides (mg/dl), median (RIQ) & $92(73-121)$ \\
\hline Homocisteyne (mg/dl), median (R|Q) & $2.08(1.7-2.8)$ \\
\hline cIMT mean (mm), median (IQR) & $0.6(0.53-0.7)$ \\
\hline Atherosclerotic plaques, $\mathrm{n}(\%)$ & $51(34.9)$ \\
\hline Bilateral plaques, $n(\%)$ & $22(15.1)$ \\
\hline \multicolumn{2}{|l|}{ Anti-rheumatic treatment } \\
\hline Synthetic DMARDs ever, n (\%) & $146(100)$ \\
\hline Methotrexate ever, n (\%) & $132(90.4)$ \\
\hline Time with methotrexate (years), median (IQR) & $4.4(1.04-9.5)$ \\
\hline Leflunomide ever, $\mathrm{n}(\%)$ & $83(56.8)$ \\
\hline Sulfasalazine ever, n (\%) & $13(8.9)$ \\
\hline Hydroxychloroquine ever, n (\%) & $99(67.8)$ \\
\hline Biological DMARDs ever, n (\%) & $76(52.05)$ \\
\hline Anti TNF $\alpha$ ever, $n(\%)$ & $47(32.2)$ \\
\hline Time with antiTNF- $\alpha$ (years), mean (SD) & $1.18(2.8)$ \\
\hline Jak inhibitor ever, $\mathrm{n}(\%)$ & $10(6.8)$ \\
\hline Anti-IL-6 ever, $\mathrm{n}(\%)$ & $28(19.2)$ \\
\hline Rituximab ever, n (\%) & 15 (10.3) \\
\hline Glucocorticoids at protocol, n (\%) & $82(56.5)$ \\
\hline Glucocorticoids dose at protocol (mg), median (IQR) & $5(0-5)$ \\
\hline
\end{tabular}

Abbreviations: RF: rheumatoid factor; ACPA: anti-citrullinated peptide antibodies, cIMT: carotid intima media thickness; $A p o B$ : apolipoprotein B, ApoA1: apolipoprotein A1, LDL: low-density lipoprotein; HDL: high-density lipoprotein; DAS28: 28-joint Disease Activity Score HAQ: Health Assessment Questionnaire; CRP: C-reactive protein; ESR: erythrocyte sedimen tation rate; IL-6: interleukin 6; Anti TNF, anti-tumor necrosis factor

Conclusion: biological disease-modifying antirheumatic drug therapy, in particu$\operatorname{lar}$ Anti TNF $\alpha$, could protect against the development of atherosclerosis in RA

Disclosure of Interests: None declared

DOI: 10.1136/annrheumdis-2021-eular.1796

\section{POS0530 HOW DOES MULTIMORBIDITY IMPACT ON THE DIRECT AND INDIRECT COSTS IN PATIENTS WITH RHEUMATOID ARTHRITIS?}

P. H. Hsieh ${ }^{1,2}$, C. Geue ${ }^{1}$, O. Wu ${ }^{1}$, E. McIntosh ${ }^{1} .{ }^{1}$ Institute of Health and Wellbeing, University of Glasgow, Health Economics and Health Technology Assessment, Glasgow, United Kingdom; ${ }^{2}$ Tri-Service General Hospital, National Defense Medical Centre, Clinical Pharmacy Department, Taipei, Taiwan, Republic of China

Background: Comorbidities are prevalent in patients with rheumatoid arthritis (RA) and associated with worse outcomes as well as higher economic burden Little is known about the impact of multimorbidity on the direct and indirect costs 
of RA. Evidence of the incremental scale of these multimorbidity costs will usefully inform RA interventions and policies.

Objectives: The aim of this study was to describe how multimorbidity impacts on the cost-of-illness, including direct and indirect costs, in patients with RA.

Methods: The Scottish Early Rheumatoid Arthritis (SERA) is a registry of patients newly presenting with RA since 2011. It contains data on patient characteristics, clinical outcomes, health-related quality of life, and employment status data. These data were linked to routinely recorded hospital admissions and primary care prescribing data. Direct costs were estimated by applying relevant unit costs to healthcare resource use quantities. Indirect cost estimates were obtained from information on employment status and hospital admissions, valued by age and sex specific wages. Two-part models (probit followed by generalized linear model) were used to estimate direct and indirect costs, adjusting for age, gender, and functional disability. The Charlson Comorbidity Index (CCI) score was calculated using patient ICD-10 diagnoses from hospital records. The number of comorbidities was categorized into "RA alone", "single comorbidity" and "multimorbidity (>1 comorbidity)".

Results: Data were available for 1,150 patients, $65.7 \%$ were female and a mean age of $57.5 \pm 14$ years. The majority of patients only had RA (54.1\%), followed by a single comorbidity (23.4\%) and multimorbidity (22.5\%). Annual total costs were significantly higher for patients with multimorbidity $(£ 6,66995 \% \mathrm{Cl} £ 4,871$ $£ 8,466$; OR $11.395 \% \mathrm{Cl} 8.14-15.87)$ and for patients with a single comorbidity (£2,075 95\% Cl £1,559-£2,591; OR 3.52 95\% Cl 2.61-4.79), when compared with RA alone (£590). The excess costs were mainly driven by direct costs ( 6,281 versus $£ 1,875$ versus $£ 556)$. Although the difference in indirect costs between patients with multimorbidity and a single comorbidity were not statistically significant ( $£ 1,218$ versus $£ 914, p=0.11$ ), patients with multimorbidity were associated with significantly higher costs than those with RA only (£594, $p<0.01$ ). Conclusion: The presence of comorbidity contributes significant excess to both direct and indirect costs among RA patients. In particular, patients with multimorbidity incurred substantially higher direct costs than those with a single comorbidity or RA only.

Acknowledgements: The study analysed the data from the Scottish Early Rheumatoid Arthritis (SERA) study with a linkage to routinely recorded health data from Information Service Division, National Service Scotland. We would like to thank all the patients, clinical and nursing colleagues who have contributed their time and support to the study, the SERA steering committee for the approval, and Allen Tervit from the Robertson Centre for Biostatistics, University of Glasgow for the timely technical supports.

Disclosure of Interests: Ping-Hsuan Hsieh: None declared, Claudia Geue: None declared, Olivia Wu Consultant of: OW has received consultancy fees from Bayer, Lupin and Takeda outside the submitted work., Emma Mclntosh: None declared

DOI: 10.1136/annrheumdis-2021-eular.1817

\section{POS0531 $\quad$ FACTORS ASSOCIATED WITH BASELINE HYPERTENSION IN EARLY RHEUMATOID ARTHRITIS: DATA FROM A REAL-WORLD LARGE INCIDENT COHORT}

B. Hadwen ${ }^{1}$, S. Stranges ${ }^{1,2,3}$, N. Klar ${ }^{1}$, K. Bindee ${ }^{4}$, J. Pope ${ }^{5}$, S. J. Bartlett ${ }^{6,7}$, G. Boire ${ }^{8}$, L. Bessette ${ }^{9}$, C. Hitchon ${ }^{10}$, G. Hazlewood ${ }^{11}$, E. Keystone ${ }^{4}$, O. Schieir ${ }^{12}$, C. Thorne ${ }^{13}$, D. Tin $^{13}$, M. F. Valois ${ }^{6}$, V. Bykerk ${ }^{14,15}$, L. Barra ${ }^{1,3,5}$ on behalf of on behalf of CATCH Investigators. ${ }^{1}$ University of Western Ontario, Epidemiology and Biostatistics, London, Canada; ${ }^{2}$ University of Western Ontario, Family Medicine, London, Canada; ${ }^{3}$ Lawson Health Research Institute, London, Canada;

${ }^{4}$ University of Toronto, Sinai Health System, Toronto, Canada; ${ }^{5}$ University of Western Ontario, Department of Medicine, London, Canada; ${ }^{6}$ McGill University, Department of Medicine, Montreal, Canada; ${ }^{7}$ Johns Hopkins University, Department of Medicine, Baltimore, United States of America; ${ }^{8}$ Université de Sherbrooke, Department of Medicine, Sherbrooke, Canada; ${ }^{9}$ Université de Laval, Department of Medicine, Quebec City, Canada; ${ }^{10}$ University of Manitoba, Department of Medicine, Winnipeg, Canada; ${ }^{11}$ University of Calgary, Department of Medicine, Calgary, Canada; ${ }^{12}$ University of Toronto, Dalla Lana School of Public Health, Toronto, Canada; ${ }^{13}$ Southlake Regional Health Center, Division of Rheumatology, Newmarket, Canada; ${ }^{14}$ Weill Cornell Medical College, Hospital for Special Surgery, New York City, United States of America; ${ }^{15}$ University of Toronto, Department of Medicine, Toronto, Canada

Background: It is not well understood why hypertension (HTN) is so common in rheumatoid arthritis (RA) patients. Reported prevalence of HTN in RA patients ranges from $4-73 \%$.(1)

Objectives: This study explored the prevalence of HTN at time of RA diagnosis and which demographic, behavioural and clinical factors were associated with HTN

Methods: Data from the Canadian Early Arthritis Cohort (CATCH), a prospective inception cohort of patients with RA $<1$ year duration, were used to analyze baseline demographic, behavioural and clinical characteristics associated with HTN, which was reported by physicians. Univariate logistic regression models were created to explore associations with baseline HTN. A multivariate logistic regression model was built based on goodness of fit indicated by likelihood ratio tests. Variables included in the model were age, sex, race, body mass index (BMI), education smoking, alcohol servings, seropositivity, disease activity and comorbidities.

Results: In total, 2052 subjects were included with mean $( \pm S D)$ age of $55( \pm 14$ years and symptom duration $5.60(5.47,5.73)$ months, $71 \%$ of subjects were female and $85 \%$ were Caucasian. HTN was reported in $26 \%$ of subjects at baseline. Hypertensive subjects were older and more likely to be male. Other factors significantly associated with HTN at baseline were lower education, ever smoking, high BMI, diabetes, hyperlipidemia, worse RA disease activity, longer duration of RA symptoms being seropositive, as well as the use of NSAIDs and/or corticosteroids (Table 1). In multivariable analysis HTN was associated with older age, overweight and obese BMI, diabetes, and hyperlipidemia. Expression of anti-citrullinated protein antibodies was inversely associated with HTN (Table 1). Other RA disease factors and treatments were not significantly associated with HTN on multivariable analysis.

Table 1. Results of univariate and multivariate logistic regression analyses exploring the association between baseline characteristics and HTN in early RA.

\begin{tabular}{|c|c|c|c|}
\hline & & $\begin{array}{l}\text { Univariate Logistic } \\
\text { Regression }\end{array}$ & $\begin{array}{l}\text { Multivariable } \\
\text { Logistic } \\
\text { Regression }\end{array}$ \\
\hline & Variable & Crude OR $(95 \% \mathrm{Cl})$ & $\begin{array}{l}\text { Adjusted OR } \\
(95 \% \mathrm{Cl})\end{array}$ \\
\hline \multirow[t]{5}{*}{ Socio-Demographic } & 20-39 years old & $0.15(0.07,0.26)$ & $0.14(0.05,0.34)$ \\
\hline & $40-59$ years old & \multicolumn{2}{|c|}{ Reference } \\
\hline & $60-79$ years old & $2.81(2.26,3.50)$ & $2.26(1.65,3.11)$ \\
\hline & $80-99$ years old & $5.87(3.36,10.25)$ & $3.80(1.53,9.41)$ \\
\hline & Female & $0.55(0.45,0.68)$ & $1.10(0.78,1.54)$ \\
\hline \multirow[t]{5}{*}{ Lifestyle/Behavioural } & $\begin{array}{l}\text { Normal weight } \\
\left(18.5-24.9 \mathrm{~kg} / \mathrm{m}^{2}\right)\end{array}$ & \multicolumn{2}{|c|}{ Reference } \\
\hline & Overweight $\left(25-29.9 \mathrm{~kg} / \mathrm{m}^{2}\right)$ & $2.33(1.74,3.11)$ & $1.63(1.10,2.43)$ \\
\hline & Obese $\left(30+\mathrm{kg} / \mathrm{m}^{2}\right)$ & $3.19(2.38,4.27)$ & $2.84(1.91,4.23$ \\
\hline & Ever-smoking & $1.41(1.15,1.73)$ & $1.02(0.75,1.40)$ \\
\hline & Post-secondary education & $0.58(0.47,0.71)$ & $0.88(0.65,1.20)$ \\
\hline \multirow[t]{8}{*}{ Clinical Characteristics } & Symptom duration & $0.99(0.99,0.99)$ & $1.00(1.00,1.00)$ \\
\hline & DAS-28 & $1.09(1.09,1.17)$ & $1.02(0.92,1.13)$ \\
\hline & ACPA+ & $0.68(0.56,0.85)$ & $0.64(0.44,0.92)$ \\
\hline & $\begin{array}{l}\text { Corticosteroid use } \\
\text { pre-baseline }\end{array}$ & $1.37(1.04,1.81)$ & Omitted \\
\hline & NSAID use at baseline & $0.68(0.55,0.84)$ & Omitted \\
\hline & Diabetes & $5.62(4.09,7.73)$ & $3.20(1.99,5.15)$ \\
\hline & Hyperlipidemia & $4.75(3.74,6.03)$ & $2.80(1.94,4.02)$ \\
\hline & CVD & $15.59(3.35,72.64)$ & Omitted \\
\hline
\end{tabular}

DAS-28; Disease activity score 28, ACPA; Anti-citrullinated protein antibody, CVD Cardiovascular disease. Pre-baseline is 29 to 365 days before entering the cohort. Baseline is within 28 days before entering the cohort. Omitted variables either failed likelihood ratio test or were colinear. Additional variables tested but found insignificant: race, alcohol servings, depression, $R F+$, and use of DMARDs.

Conclusion: Approximately 1 in 4 diagnosed with RA had HTN reported by their rheumatologists, which is similar to that of the general population. This suggests that increased risk of HTN in RA patients may develop as RA disease or treatment time progresses. Factors that may be predictive of this excess risk will be explored in further analysis.

REFERENCES:

[1] Panoulas VF, Metsios GS, Pace AV, et al. Hypertension in rheumatoid arthritis. Rheumatology (Oxford) 2008;47:1286-98.

Acknowledgements: The CATCH study was designed and implemented by the investigators and financially supported through unrestricted research grants from: Amgen and Pfizer Canada - Founding sponsors since January 2007; AbbVie Corporation and Hoffmann-LaRoche since 2011; Medexus Inc. since 2013;, Merck Canada since 2017, Sandoz Canada, Biopharmaceuticals since 2019, Gilead Sciences Canada since 2020 and Fresenius Kabi Canada Ltd. since 2021. Previously funded by Janssen Biotech from 2011-2016, UCB Canada and Bristol-Myers Squibb Canada from 2011-2018, Sanofi Genzyme from 2016-2017, and Eli Lilly Canada from 2016-2020.

Disclosure of Interests: Brook Hadwen: None declared, Saverio Stranges: None declared, Neil Klar: None declared, Kuriya Bindee: None declared, Janet Pope Speakers bureau: UCB, Consultant of: AbbVie, Actelion, Amgen, Bayer, BMS Eicos Sciences, Eli Lilly \& Company, Emerald, Gilead, Janssen, Merck, Novartis Pfizer, Roche, Sandoz, Sanofi, UCB;, Grant/research support from: Abbvie, BMS, Eli Lilly \& Company, Merck, Roche, Seattle Genetics, UCB, Susan J. Bartlett Consultant of: Pfizer, UCB, Lilly, Novartis, Merck, Janssen, Abbvie, Gilles Boire Speakers bureau: Merck, BMS, Pfizer, Janssen, Grant/research support from: Amgen, Abbvie, BMS, Eli Lilly, Merck, Novartis, Pfizer, Sandoz, Louis Bessette Speakers bureau: Amgen, BMS, Janssen, Roche, UCB, AbbVie, Pfizer, Merck, Celgene, Sanofi, Lilly, Novartis, Consultant of: Amgen, BMS, Janssen, Roche, UCB, AbbVie, Pfizer, Merck, Celgene, Sanofi, Lilly, Novartis., Grant/research 